\section{IN THE NEWS}

Gulf-war vaccination

'...classified 'secret' injections [given to soldiers before deployment in the 1991 Gulf War]... have most probably led to the development of autoimmune-induced osteoporosis.'

This is the claim made in a leaked report for the war pensions agency by Lieutenant Colonel Graham Howe, clinical director of psychiatry with the British forces health senvice in Germany (The Times). This is the first official support for such a link.

The B ritish Ministry of Defence (MoD) has rigorously denied the existence of a specific Gulf war syndrome -

‘...Gulf veterans do not present with a distinct and identifiable pattern of symptoms or signs.' (MoD website). For example, veterans have complained of a range of symptoms, from respiratory and digestive problems to memory and psychological problems (Reuters). Backed up by independent studies - such as a report by the Medical Research Council in May 2003 (BBC News) - the MoD also refutes any link between illness and vaccination.

However, Lt Col Howe describes the case of LanceCorporal Alex Izett, who received vaccines against anthrax, botulism, plague and other unspecified biological agents in anticipation of war, but was not eventually required to fight. As the subject was not involved in fighting, the report states that the common factor linking the osteoporosis with which he suffers to the high incidence of osteoporosis in Gulf-war veterans is the vaccines he received.

Much of the press coverage has focused on the 'cocktail' of vaccines that soldiers are thought to have received, with some reports that 28 'jabs' were given. The coverage has been sufficient for this controversial issue to be raised in the House of Lords by Labour peer Lord Morris of Manchester (The Guardian).

Kirsty M inton

\title{
BCR and TLR9 cooperation in autoimmunity
}

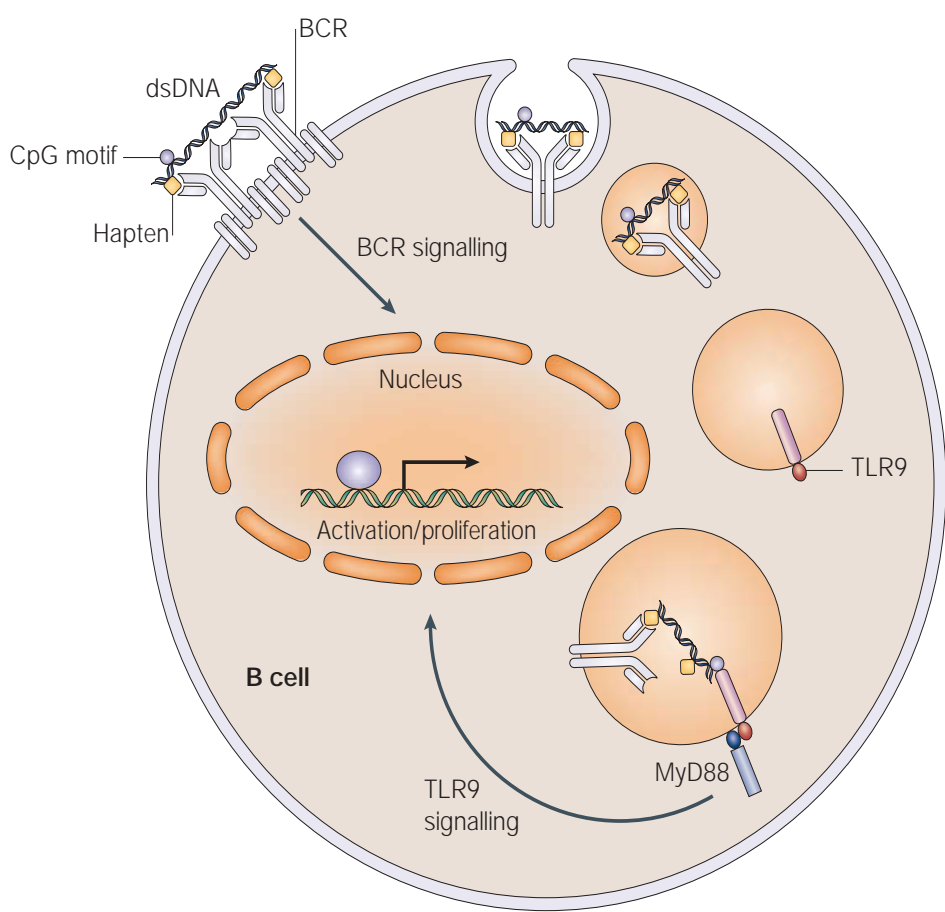

The binding of haptenated $\mathrm{CpG}$ double-stranded (ds)DNA to hapten-specific B cells induces a suboptimal signal from a minimally crosslinked B-cell receptor $(B C R)$. The $B C R$ also functions to shuttle the CpG DNA antigen to an intracellular compartment containing Toll-like receptor 9 (TLR9). TLR9 signalling through a MyD88-dependent pathway, together with the signal from the BCR, leads to B-cell activation and proliferation.

\section{STAT3 - a potential target for tumour immunotherapy}

Inhibitors of signal transducer and activator of transcription 3 (STAT 3) could be used to activate immuneresponses against tumours, according to a report now published in Nature Medicine.

Although tumour growth can activate inflammatory responses, theimmunesystem generally ignores established cancers. AsSTAT 3 is constitutively activated in many cancers, Wang et al. decided to investigate whether activity of this transcription factor could be involved in blocking theinitiation of antitumour immuneresponses.
First, theauthors observed that disruption of STAT 3 signalling led to a selective increase in the production of pro-inflammatory mediators, including tumour-necrosis factor, interleukin-6 (IL-6) and the chemokine C C 5 by tumour-cell lines. By contrast, constitutive expression of STAT 3 led to the inhibition of cytokineand chemokine production by tumour cells.

Next, they studied the effect of the pro-inflammatory mediators that are produced by STAT 3-disrupted tumours on cells of theimmune system. Cells of theinnateimmune
Systemic lupus erythematosus (SLE) and other autoimmune diseases often involve a response to immune complexes containing DNA or chromatin-associated proteins. A new study published in Immunity has now set out to explain how the autoreactive $B$ cells involved are activated and the exact nature of their specificity.

The AM 14 autoimmune mouse strain produces $B$ cellsthat recognize autologous IgG2a specific for DNA. In particular, thel gG2a antibodies that are recognized can complex with chromatin released from dying cells to form multivalent DNA-containing immunecomplexes (chromatin-ICS). Previous experiments haveshown that in addition to $B$-cell receptor ( $B C R$ ) recognition of IgG2a, the DNA portion of chromatin-ICs is involved in the activation of AM 14 B cells through Toll-like receptor 9 (TLR9). TLR9 recognizes hypomethylated $\mathrm{CpG}$ containing bacterial DNA, but such motifs arerare in mammalian DNA. Theauthors set out to investigatethis system further using a series of defined haptenated double-stranded DNA fragments (such as NIP2-CG50-NIP) recognized by anti-hapten (NIPspecific) antibodies.

First, what are the relative roles of TLR9 and BCR signalling? Anti-NIP-NIP2-CG50-NIP immune

system, including macrophages and neutrophils, were activated to producefurther pro-inflammatory mediators after exposure to the supernatant from tumour cells in which STAT 3 was blocked. Furthermore, this supernatant also activated dendritic cells (DCs), which were then ableto stimulatetumourantigen-specific naive T cellsto proliferateand produce IL-2 and interferon- $\gamma$. These results show that blocking STAT 3 signalling in tumour cells results in theactivation of both innate and adaptive anti-tumour immuneresponses.

Further experiments looked at the effects of constitutive expression of STAT 3 by tumour cells. In addition to inhibiting the production of pro-inflammatory mediators by thesecells, the authors showed that constitutiveSTAT 3 activity induced tumour cellsto 
H I G H L I G H T S

complexes stimulated AM 14 B-cell proliferation, but neither antibody nor DNA fragment alone was able to do so. Blocking signalling through TLR9 inhibited this response. However, stimulation with DNA fragments plusan IgG2a antibody that does not form immunecomplexes with DNA did not lead to B-cell activation, indicating that independent ligation of cellular receptors by monovalent antibody and DNA is not sufficient for activation. The authors speculated that BCR-mediated uptake of DNA facilitates stimulation of intracellular TLR9, but it was unclear whether the BCR was acting as a passive shuttle or whether BCR crosslinking had a role in B-cell activation. Using a monosubstituted DNA fragment (NIP-CG50) that is unable to form multivalent immunecomplexes, it was shown that $B C R$ crosslinking is not essential for $B$-cell activation in this system. However, signals from the BCR do contribute to stimulation by chromatin-ICs from dying cells, which have alower frequency of CPG motifs than fragment-ICS, as shown by the partial inhibition by cyclosporin A. It seems that signalsfrom both theBCR and TLR9 combineto givean optimal response.

Second, what is the precise ligand that is recognized by TLR9? Immune complexesformed from threetypes of haptenated DNA fragment that differ in terms of immunostimulatory $\mathrm{CpG}$ content werecompared, and only the CG50 fragments, which contain the highest density of canonical immunostimulatory CpG motifs, induced a strong B-cell proliferativeresponse. M ethylation of the DNA completely blocked its stimulatory capacity. Therefore, the rarehypomethylated DNA motifs found in mammalian DNA, which the authors suggest might be enriched in apoptotic chromatin, arean essential component of theseimmunecomplexes.

However, for chromatin-ICs to form in vivo and trigger disease, $B$ cells must first produce DNAspecific antibodies. I mportantly, the authors show that the proposed model of BCR/TLR 9 co-operation can be extended to DNA-specific B cells (see figure). They suggest that thismight also be a general mechanism for the recognition of opsonized foreign antigens such as bacterial DNA.

Kirsty M inton

(2) References and links ORIGINAL RESEARCH PAPER Viglianti, G. A. et al. Activation of autoreactive $B$ cells by $C p G$ dsDNA. Immunity 19, 837-847 (2003) FURTHER READING Shlomchik, M. J . et al. From $T$ to $B$ and back again: positive feedback in systemic autoimmune disease. Nature Rev. Immunol. 1, 147-153 (2001)

\section{produce a diverserange of factors, including I L-10 and vascular endothelial growth factor, that inhibited DC maturation. Interestingly, a common point of convergence of theinhibitors of immuneactivation was the induction of STAT 3 in the DCs themselves, indicating a cascade of STAT 3 activation first in thetumour and then in the DCs. \\ Thisstudy highlights therole of STAT 3 in regulating innateand adaptiveimmuneresponses to tumours, and, as the authors conclude, indicates that blocking STAT 3 signalling with specific inhibitors could have direct anti- tumour effects.}

Jenny Buckland

(D) References and links ORIGINAL RESEARCH PAPER Wang, T. et al. Regulation of the innate and adaptive immune responses by Stat- 3 signaling in tumor cells. Nature Med. 10, 48-54 (2004)

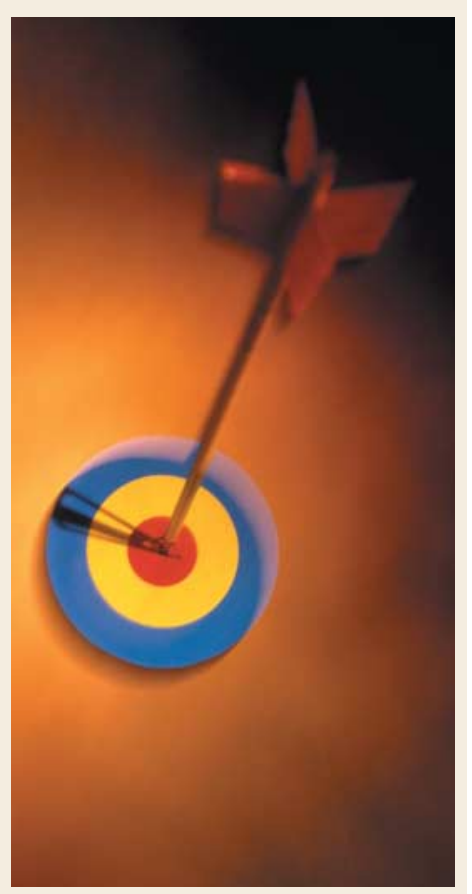

\section{IN BRIEF}

\section{T-CELL ACTIVATION}

Evidence that structural rearrangements and/or flexibility during TCR binding can contribute to T cell activation.

Krogsgaard, M. et al. Mol. Cell 12, 1367-1378 (2003)

Although the ability of a peptide to activate $\mathrm{T}$ cell often correlates with the half-life of the T-cell-receptor (TCR)-peptide-M HCcomplex interaction, not all peptides conform to this rule. Krogsgaard et al. studied peptideanalogues of a moth cytochromec ( $\mathrm{MCC}$ ) peptide, two of which - K2 and K3 - weremore stimulatory than predicted. Thermodynamic analysis of the TCR-peptide-M HC interaction indicated greater changes in heat capacity for K 2 and K 3, compared with M CC. These changes were associated with structural rearrangement and flexibility after TCR binding. By considering both thehalf-life of TCR binding and the change in heat capacity, theauthors could predict the ability of a peptide to activate a T cell, indicating that conformational change and flexibility contribute to $\mathrm{T}$-cell activation.

\section{AUTOIMMUNITY}

Autoimmunity is triggered by cPR-3 (105-201), a protein complementary to human autoantigen proteinase-3.

Pendergraft III, W. F. et al. Nature Med. 10, 72-79 (2004)

Antibodies specific for neutrophil granuleantigens, including proteinase 3 (PR3), are associated with autoimmune vasculitis. Theauthors of this paper detected antibodies specific for complementary PR3 (CPR3) - a peptidetranslated from theantisenseDNA strand of thePR3 gene - in the sera of several patients with vasculitis with PR3-specific antibodies, but not in those without PR3-reactiveantibodies or heal thy individuals. The CPR3and PR3-specific antibodies did not cross-react with thesame antigen but formed an idiotypic pair. Mice immunized with human recombinant CPR3 developed both CPR3- and PR3-specific antibodies, indicating that peptidestranslated from theanti-sense strand of an autoantigen, or microbial-derived peptides that mimic this complementary peptide, could initiate autoimmunity.

\section{LEUKOCYTE SIGNALLING}

Analysis of C 5 a-mediated chemotaxis by lentiviral delivery of small interfering RNA.

Hwang, J. I. et al. Proc. Natl Acad. Sci. USA 101, 488-493 (2004)

The authors used small interfering RNA to establish the role of specific components of the intracellular heterotrimeric G-protein complex in the signalling pathway that mediates chemotaxis. Knockdown of the $\beta \beta 2$ subunit in the mouse macrophage-cell line J74A.1 abolished migration towards C 3 a and C5a, whereas cells lacking other $\mathrm{G} \beta$ or $\mathrm{G} \alpha$ subunits migrated normally. G $\beta 2$ knockdown diminished the $\mathrm{C} 5 \mathrm{a}$-induced $\mathrm{Ca}^{2+}$ signal and eliminated sustained Akt phosphorylation, providing evidence that phospholipase $C \beta$ and $A k t$ are downstream effectors of this signalling pathway. However, in the absence of $G \beta 2$, levels of both $\mathrm{G} \gamma 2$ and $\mathrm{G} \gamma 5$ were downregulated, indicating that further studies are required to define the precise molecular mechanisms by which $\mathrm{G} \beta 2$ regulates $\mathrm{C} 5 \mathrm{a}$-induced chemotaxis. 